University of Wollongong

Research Online

Faculty of Engineering and Information

Faculty of Engineering and Information

Sciences - Papers: Part B

Sciences

2017

\title{
A Unified Robust Motion Controller Design for Series Elastic Actuators
}

Emre Sariyildiz

University of Wollongong, emre@uow.edu.au

Gong Chen

National Univeristy of Singapore

Haoyong Yu

National Univeristy of Singapore

Follow this and additional works at: https://ro.uow.edu.au/eispapers1

Part of the Engineering Commons, and the Science and Technology Studies Commons

Research Online is the open access institutional repository for the University of Wollongong. For further information contact the UOW Library: research-pubs@uow.edu.au 


\title{
A Unified Robust Motion Controller Design for Series Elastic Actuators
}

\author{
Abstract \\ Series Elastic Actuators (SEAs) have several mechanical superiorities over conventional stiff and non- \\ back-drivable actuators, e.g., lower reflected inertia at output, greater shock tolerance, low cost force \\ measurement, energy storage, safety, and so on. However, their applications generally suffer from \\ performance limitations, particularly in position control, due to insufficient controller designs. This paper \\ proposes a unified Active Disturbance Rejection (ADR) motion controller for the robust position and force \\ control problems of SEAs by using Differential Flatness (DF) and Disturbance Observer (DOb). It can \\ suppress not only matched but also mismatched disturbances. Robust state and control input references \\ are systematically generated in terms of a fictitious design variable, namely differentially flat output, \\ estimations of disturbances, and their successive time derivatives. The proposed robust motion controller \\ improves the performance of SEAs when they suffer from internal and external disturbances, such as \\ friction, inertia variation and external load, in real implementations. Experimental results are given to \\ validate the proposal. \\ Disciplines \\ Engineering | Science and Technology Studies \\ Publication Details \\ Sariyildiz, E., Chen, G. \& Yu, H. (2017). A Unified Robust Motion Controller Design for Series Elastic \\ Actuators. IEEE/ASME Transactions on Mechatronics, 22 (5), 2229-2240.
}

This journal article is available at Research Online: https://ro.uow.edu.au/eispapers1/748 


\title{
A Unified Robust Motion Controller Design for Series Elastic Actuators
}

\author{
Emre Sariyildiz, Member, IEEE, Gong Chen, and Haoyong Yu, Member, IEEE
}

\begin{abstract}
Series Elastic Actuators (SEAs) have several mechanical superiorities over conventional stiff and non-backdrivable actuators, e.g., lower reflected inertia at output, greater shock tolerance, low cost force measurement, energy storage, safety, and so on. However, their applications generally suffer from performance limitations, particularly in position control, due to insufficient controller designs. This paper proposes a unified Active Disturbance Rejection (ADR) motion controller for the robust position and force control problems of SEAs by using Differential Flatness (DF) and Disturbance Observer (DOb). It can suppress not only matched but also mismatched disturbances. Robust state and control input references are systematically generated in terms of a fictitious design variable, namely differentially flat output, estimations of disturbances and their successive time derivatives. The proposed robust motion controller improves the performance of SEAs when they suffer from internal and external disturbances, such as friction, inertia variation and external load, in real implementations. Experimental results are given to validate the proposal.
\end{abstract}

Index Terms-Disturbance Rejection, Differential Flatness, Disturbance Observer, Motion Control, Series Elastic Actuator.

\section{INTRODUCTION}

$\mathrm{H}$ IGH precision position control and repeatability are the top priorities of many conventional robot applications [1-3]. In order to improve the robustness, stability and performance of position control, robots are designed by using stiff and nonback-drivable mechanical components [4]. However, several studies have showed that accurate position control is not sufficient to perform fine motion tasks in which robots interact with environments, e.g., grinding and polishing [5-7]. Although explicit and implicit force control methods have been proposed to perform fine motion tasks by actively adjusting the compliance of stiff robots, they suffer from low performance, stability and safety problems in practice [8-11]. For example, industrial robots cannot work alongside humans and are often kept in human-free cages in a manufacturing plant due to safety problems $[12,13]$.

In the new era of robotics, physical interaction with unstructured and dynamic environments becomes a more

Manuscript received August 30, 2016; revised November 1, 2016, January 3, 2017, March 12, 2017 and May 15 2017; accepted June 3, 2017. This work was supported in part by the Singapore Ministry of Education Academic Research Fund Tier 1, under the WBS No. R-397-000-218-112, in part by the Defense Innovative Research Programme, MINDEF of Singapore under Grant No. MINDEF-NUS-DIRP/2012/02, and in part by the Ministry of Education of Singapore Tier 2 Grant under the grant No. MOE2014-T2-1-115. dominant requirement for many advanced robot applications, e.g., rehabilitation, humanoids and human-robot collaboration [5-7]. Against traditional robotics "stiffer is better" rule of thumb, passive compliance control, in which robots have intrinsic compliant elements, has been proposed to overcome the fundamental performance, stability and safety problems of conventional stiff robots [14-16]. Series Elastic Actuators (SEAs) are the most famous inherently compliant actuation systems which consist of a spring in series with a stiff actuator [15]. They have several mechanical superiorities over conventional stiff actuators in force control, e.g., lower mechanical output impedance, greater tolerance to impact load, higher force fidelity, and so on $[15,16]$. However, the motion control problem of compliant robots is more complicated than that of conventional stiff robots, particularly in position control. For example, the vibration at link-side may degrade the performance or even damage the robot as the speed of tasks is increased; and external disturbances may directly degrade the position accuracy at link-side due to the sensitivity problem [17, 18]. In order to utilize the mechanical superiorities of SEAs in advanced robot applications, their position and force control performances should be improved.

Several studies have been conducted to improve the position control performance of compliant robots. However, they have limitations in real implementations of SEAs. Singular perturbation method separately controls slow and fast states of compliant robots, but it is only applicable if the stiffness is relatively large, the dynamic model is precisely known, and the external disturbances are negligible $[19,20] . \mu$-synthesis-based adaptive robust control is applied to a linear motor when it suffers from relatively high stiffness of ball bearings in high frequencies [21]. In general, PID controllers are designed at motor-side, and the link-side's position accuracy is improved by using feed-forward control. It is sensitive to disturbances at link-side such as load and is applicable for only regulation [2224]. Intelligent and advanced robust controllers have been implemented to improve the position control performance of compliant robots, but, in general, they are computationally demanding and very complex for real-time implementations [25-27]. Time-Delay Estimation (TDE) scheme was applied to

E. Sariyildiz is with the School of Mechanical, Materials, Mechatronic and Biomedical Engineering, University of Wollongong, Wollongong, NSW, Australia 2522. (e-mail: emre@uow.edu.au).

G. Chen and H. Yu are with the Department of Biomedical Engineering, National University of Singapore, 4 Engineering Drive 3, \#04-08, Singapore, 117583. (e-mail: chen.gong@u.nus.edu, bieyhy@nus.edu.sg). 
improve the robustness and link-side position accuracy of SEAs in [18]. Simulation results of "COMAN" were given by neglecting practical design constraints such as noise-sensitivity $[18,28]$. Resonance Ratio Control was applied to the robust position control problem of SEAs in [17]. It suppresses the vibration at link-side by degrading the robustness at motor-side. Arm-DOb is proposed to improve the robustness, but it increases the analysis and design complexity and can suppress only constant external disturbances in practice.

Force/impedance control of SEAs has been widely studied in the last two decades. Conventionally, it is performed by using a single-loop PID force controller that is combined with a feedforward controller $[4,16]$. It is sensitive to internal disturbances; the stability and performance of force control may deteriorate if nonlinear disturbances, such as friction, are not negligible [29]. A cascade motion controller, which has inner velocity and outer force control loops, was proposed to improve the force control stability of SEAs [29, 30]. Its performance is limited by control gains and may deteriorate by internal disturbances in practice. The robustness of the cascade motion controller is improved by using a DOb that is designed for the velocity control inner-loop [31-33]. The well-known causality constraint of the observer design is satisfied by using a high order low-pass filter (LPF) [28, 31]. The analysis and design of a high order LPF of DOb are not straightforward in classical control approach. The robust stability and performance of the force controller should be further investigated by considering conservatism due to high order LPF [28]. For example, unexpected stability problem of the robust force controller was reported in [34]. Resonance Ratio Control was similarly applied to the robust force control problem of SEAs in [17].

In this paper, a unified ADR motion controller is proposed for SEAs in state space. This controller can be tuned to perform either position or force control applications. Modern control theory can provide many convenience methods to adjust the stability and performance of SEAs; e.g., the vibration at linkside can be simply suppressed by placing all poles of the system on the real axis via state feed-back control. However, it requires precise dynamic model of the system and is sensitive to external disturbances. Moreover, trajectory tracking controller design is not straightforward in modern control; i.e., not only control input but also state references should be adequately generated. To utilize modern control methods in the real motion control implementations of SEAs, the robust state and control input references of a state feed-back controller are systematically generated by combining DF and DOb in state space.

In the first step, the dynamic model of an SEA is derived by using the analogy of a two-mass-spring-damper system in state space. This system suffers from not only matched but also mismatched disturbances. Then, a state feed-back controller is designed for the nominal plant model by neglecting the disturbances so that the nominal stability and performance of the motion control system are adjusted. Lastly, the robust state and control input references of the state feed-back controller are systematically generated in terms of differentially flat output variable, estimations of disturbances and their successive time derivatives by using DF and DOb. The former is used to

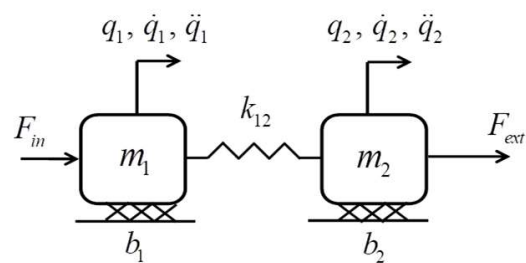

a) Free motion.

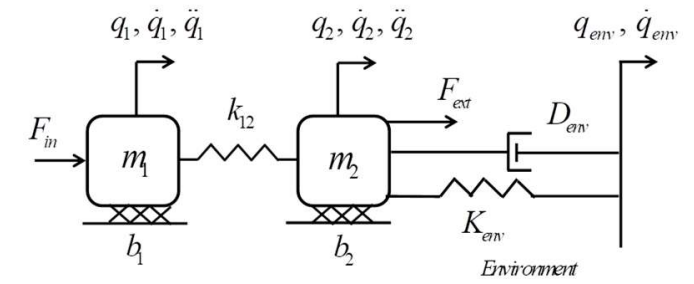

b) Contact motion.

Fig.1: Model of a Series Elastic Actuator.

perform trajectory tracking control in state space. The latter is used to achieve nominal performance by suppressing/ cancelling disturbances of SEAs in practice.

The proposed unified motion controller has a two-degreesof-freedom (2-DOF) control structure, thus its performance and robustness can be independently adjusted by tuning the state feed-back controller and $\mathrm{DOb}$, respectively. It significantly improves the stability and performance by suppressing the internal and external disturbances of SEAs such as friction, inertia variation, load and un-modeled dynamics. The validity of the proposed robust motion controller is verified by giving position and force control experimental results of an SEA.

The rest of the paper is organized as follows. In section II, the dynamic model of an SEA is derived. In section III, a second order $\mathrm{DOb}$ is proposed to estimate disturbances and their successive time derivatives. In section IV, a new theorem is proposed to design a robust trajectory tracking controller in state space. In section $\mathrm{V}$, it is applied to the robust position and force control problems of an SEA. In section VI, experimental results are given. The paper ends with conclusion given in the last section.

\section{Model of A SERIEs Elastic ACtUAtor}

A simple yet accurate lumped parameter dynamic model of an SEA can be derived by using the analogy of a two-massspring-damper system that is shown in Fig. 1. In this figure, $m_{1}$ and $m_{2}$ represent the first and second masses, respectively, e.g., the former represents the masses of motor and gear box, and the latter represents the masses of link and load; $b_{i}$ represents the $i^{\text {th }}$ viscous friction coefficient; $q_{i}, \dot{q}_{i}$ and $\ddot{q}_{i}$ represent the position, velocity and acceleration of the $i^{\text {th }}$ mass, respectively; $q_{e n}$ and $\dot{q}_{e n v}$ represent the position and velocity of environment, respectively; $k_{12}$ represents the stiffness of the spring between the first and second masses; $F_{i n}$ and $F_{\text {ext }}$ represent the input and external forces, i.e., motor torque and external load, respectively; and $D_{e n v}$ and $K_{e n v}$ represent environmental damping and stiffness, respectively.

The position and force control systems are illustrated as free and contact motions in Fig. 1a and Fig. 1b, respectively. The former's goal is defined as tracking the desired position 
trajectory of the actuator's link, i.e., $q_{2}^{\text {des }}$. However, the latter's goal is defined as tracking the desired force trajectory of the actuator's spring, i.e., $F_{\text {spring }}^{d k s}=k_{12}\left(q_{1}-q_{2}\right)^{d e s}$. The force control goal can also be considered as the desired deflection of the spring. It is one of the main superiorities of SEAs over conventional stiff actuators in force control.

The dynamic equations of an SEA can be directly derived from Fig.1 as follows:

$$
\begin{aligned}
& m_{1 n} \ddot{q}_{1}+b_{1 n} \dot{q}_{1}=F_{i n}-k_{12 n}\left(q_{1}-q_{2}\right)-\tau_{\text {dis }}^{\text {matched }} \\
& m_{2 n} \ddot{q}_{2}+b_{2 n} \dot{q}_{2}=k_{12 n}\left(q_{1}-q_{2}\right)-\tau_{\text {dis }}^{\text {mismatched }}
\end{aligned}
$$

where $m_{\bullet n}, b_{\bullet n}$ and $k_{12 n}$ represent the nominal parameters of $m_{\bullet}, b_{\bullet}$, and $k_{12}$, respectively;

$$
\tau_{\text {dis }}^{\text {matched }}=\left(m_{1}-m_{1 n}\right) \ddot{q}_{1}+\left(k_{12}-k_{12 n}\right)\left(q_{1}-q_{2}\right)+f_{\text {frc } 1}+f_{\text {unm } 1}
$$

represents the matched disturbances at motor-side;

$$
\tau_{\text {dis }}^{\text {mismatched }}=\left\{\begin{array}{lc}
\tau_{\text {dism }}^{\text {mitched }} & \text { Free Motion } \\
\tau_{\text {dism }}^{\text {mismatched }} & \text { Contact Motion }
\end{array}\right.
$$

in which $\tau_{\text {dis } \operatorname{nismatched}}^{\text {ind }}=F_{\text {ext }}+\left(m_{2}-m_{2 n}\right) \ddot{q}_{2}+\left(k_{12 n}-k_{12}\right)\left(q_{1}-q_{2}\right)+f_{\text {frc } 2}+f_{\text {umm } 2}$ represents the mismatched disturbances at link-side in free motion, and $\tau_{\text {dis } C M}^{\text {nimatched }}=\tau_{\text {dis } F M}^{\text {nismatched }}+D_{e n v}\left(\dot{q}_{2}-\dot{q}_{e n v}\right)+K_{e n v}\left(q_{2}-q_{e n v}\right)$ represents the mismatched disturbances at link-side in contact motion; $f_{\text {frc } 1}$ and $f_{\text {frc } 2}$ represent disturbances due to frictions, such as Coulomb and Stribeck frictions, at motor and link sides, respectively [5]; and $f_{\text {uuml }}$ and $f_{\text {uum } 2}$ represent disturbances due to any linear and nonlinear un-modeled dynamics, such as backlash, at motor and link sides, respectively.

Without any approximation, Eq. (1) can be rewritten in state space as follows:

$$
\dot{\mathbf{x}}=\mathbf{A}_{\mathbf{n}} \mathbf{x}+\mathbf{b}_{\mathbf{n}} u-\boldsymbol{\tau}_{\text {dis }}
$$

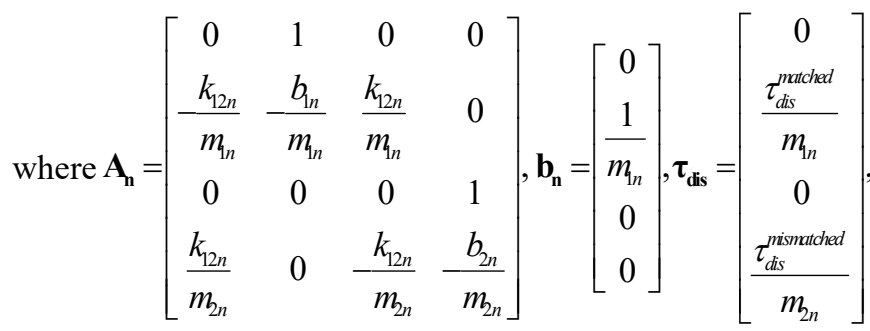

$\mathbf{x}=\left[\begin{array}{llll}q_{1} & \dot{q}_{1} & q_{2} & \dot{q}_{2}\end{array}\right]^{T}$ and $u=F_{i n}$.

Equation (4) shows that all states of an SEA can be controlled by using a state feed-back controller. It can be easily verified by showing that the controllability matrix of Eq. (4) is full rank. It is shown in section IV that controllability is the necessary and sufficient condition to design the proposed robust trajectory tracking controller.

Equation (4) also shows that the dynamic model of an SEA includes matched and mismatched disturbances in the second and fourth channels, respectively. The former can be directly cancelled by feeding-back its estimation through control input. However, the latter cannot be similarly cancelled since there is no control input in its channel. Therefore, an advanced ADR motion controller should be designed to achieve the robustness of SEAs [35-37].

\section{Disturbance OBSERVER}

$\mathrm{A} \mathrm{DOb}$ is an ADR control tool that is widely used to estimate plant uncertainties, external disturbances and their successive derivatives [35, 38]. In Section V, robust position and force controllers are proposed for SEAs by using the estimations of the disturbance vector and its first and second order derivatives. They are obtained by using a second order DOb as follows:

In the design of a DOb, the dynamic characteristics of disturbances should be predetermined by using some assumptions. For example, conventional DOb is designed by assuming constant disturbances. However, it can estimate not only constant but also variable disturbances if they stay within the bandwidth of DOb [38]. In order to design the second order $\mathrm{DOb}$, let us assume that the third order derivative of the disturbance vector is zero, i.e., $\dddot{\boldsymbol{\tau}}_{\mathrm{dis}} \cong \mathbf{0}$.

Let us first design auxiliary variable vectors in terms of the disturbance vector, its first and second order derivatives and the state vector of the system which is given in Eq. (4).

$$
\begin{aligned}
& \mathbf{z}_{1}=\boldsymbol{\tau}_{\text {dis }}+L_{1} \mathbf{x} \\
& \mathbf{z}_{\mathbf{2}}=\dot{\boldsymbol{\tau}}_{\text {dis }}+L_{2} \mathbf{x} \\
& \mathbf{z}_{\mathbf{3}}=\ddot{\boldsymbol{\tau}}_{\text {dis }}+L_{3} \mathbf{x}
\end{aligned}
$$

where $\mathbf{z}_{\mathbf{i}} \in R^{4}$ represents the $i^{\text {th }}$ auxiliary variable vector; $L_{i} \in R$ represents the $i^{\text {th }}$ gain of DOb; and $\dot{\boldsymbol{\tau}}_{\text {dis }}$ and $\ddot{\boldsymbol{\tau}}_{\text {dis }} \in R^{4}$ represent the first and second order derivatives of the disturbance vector, $\tau_{\text {dis }}$.

Time derivatives of Eq. (5-7) are derived as follows:

$$
\begin{aligned}
& \dot{\mathbf{z}}_{1}=-L_{1} \mathbf{z}_{1}+\mathbf{z}_{2}+L_{1}\left(\mathbf{A}_{\mathbf{n}} \mathbf{x}+\mathbf{b}_{\mathbf{n}} u+L_{1} \mathbf{x}\right)-L_{2} \mathbf{x} \\
& \dot{\mathbf{z}}_{2}=-L_{2} \mathbf{z}_{1}+\mathbf{z}_{3}+L_{2}\left(\mathbf{A}_{\mathbf{n}} \mathbf{x}+\mathbf{b}_{\mathbf{n}} u+L_{1} \mathbf{x}\right)-L_{3} \mathbf{x} \\
& \dot{\mathbf{z}}_{3}=-L_{3} \mathbf{z}_{1}+L_{3}\left(\mathbf{A}_{\mathbf{n}} \mathbf{x}+\mathbf{b}_{\mathbf{n}} u+L_{1} \mathbf{x}\right)
\end{aligned}
$$

Since the nominal parameters of the system and control input are known and system sates are measured, the estimations of the auxiliary variable vectors can be simply obtained by substituting them into Eq. (8-10) as follows:

$$
\begin{aligned}
& \dot{\hat{\mathbf{z}}}_{1}=-L_{1} \hat{\mathbf{z}}_{1}+\hat{\mathbf{z}}_{2}+L_{1}\left(\mathbf{A}_{\mathbf{n}} \mathbf{x}+\mathbf{b}_{\mathbf{n}} u+L_{1} \mathbf{x}\right)-L_{2} \mathbf{x} \\
& \dot{\hat{\mathbf{z}}}_{2}=-L_{2} \hat{\mathbf{z}}_{1}+\hat{\mathbf{z}}_{3}+L_{2}\left(\mathbf{A}_{\mathbf{n}} \mathbf{x}+\mathbf{b}_{\mathbf{n}} u+L_{1} \mathbf{x}\right)-L_{3} \mathbf{x} \\
& \dot{\mathbf{z}}_{3}=-L_{3} \hat{\mathbf{z}}_{1}+L_{3}\left(\mathbf{A}_{\mathbf{n}} \mathbf{x}+\mathbf{b}_{\mathbf{n}} u+L_{1} \mathbf{x}\right)
\end{aligned}
$$

where $\hat{\mathbf{z}}_{\mathbf{i}} \in R^{4}$ represents the estimation of the $i^{\text {th }}$ auxiliary variable vector, $\mathbf{z}_{\mathbf{i}}$.

Let us define the error vectors of the auxiliary variable estimation by using $\mathbf{e}_{1}=\mathbf{z}_{1}-\hat{\mathbf{z}}_{1}, \mathbf{e}_{2}=\mathbf{z}_{2}-\hat{\mathbf{z}}_{2}$ and $\mathbf{e}_{3}=\mathbf{z}_{3}-\hat{\mathbf{z}}_{3} \in R^{4}$. The dynamic equation of the auxiliary variable estimation error is derived by subtracting Eq. (11-13) from Eq. (8-10) respectively as follows:

$$
\dot{\mathbf{e}}(t)=\Psi \mathbf{e}(t)
$$

where $\mathbf{e}(t)=\left[\begin{array}{c}\mathbf{e}_{1} \\ \mathbf{e}_{2} \\ \mathbf{e}_{3}\end{array}\right], \dot{\mathbf{e}}(t)=\left[\begin{array}{c}\dot{\mathbf{e}}_{1} \\ \dot{\mathbf{e}}_{2} \\ \dot{\mathbf{e}}_{3}\end{array}\right], \boldsymbol{\Psi}=\left[\begin{array}{lll}-L_{1} \mathbf{I}_{4} & \mathbf{I}_{4} & \mathbf{0}_{4} \\ -L_{2} \mathbf{I}_{4} & \mathbf{0}_{4} & \mathbf{I}_{4} \\ -L_{3} \mathbf{I}_{4} & \mathbf{0}_{4} & \mathbf{0}_{4}\end{array}\right]$, and $\mathbf{I}_{4}$ and $\mathbf{0}_{4}$

represent $4 \times 4$ identity and null matrices, respectively.

The eigenvalues of $\boldsymbol{\Psi}$ are derived by solving 


$$
\operatorname{det}\left(\lambda \mathbf{I}_{12}-\boldsymbol{\Psi}\right)=\left(\lambda^{3}+L_{1} \lambda^{2}+L_{2} \lambda+L_{3}\right)^{4}=0
$$

Equation (14) and Eq. (15) show that $\mathbf{e} \rightarrow 0$ asymptotically if the gains of DOb are properly tuned, i.e., all eigenvalues of $\boldsymbol{\Psi}$ are negative. Larger magnitude eigenvalues correspond to faster estimations of auxiliary variable vectors.

It is noted that the assumption of $\dddot{\boldsymbol{\tau}}_{\mathrm{dis}} \cong \mathbf{0}$ is not crucial in the design of the proposed second order DOb. When $\dddot{\boldsymbol{\tau}}_{\text {dis }} \neq \mathbf{0}$, the error dynamics can be rewritten as follows:

$$
\dot{\mathbf{e}}(t)=\boldsymbol{\Psi e}(t)+\left[\begin{array}{c}
\mathbf{0}_{4 \times 1} \\
\mathbf{0}_{4 \times 1} \\
\dddot{\boldsymbol{\tau}}_{d i s}(t)
\end{array}\right]_{12 \times 1}
$$

One can easily show by analytically solving Eq. (16) that it is input to state stable if the observer gains are adequately tuned and $\dddot{\boldsymbol{\tau}}_{d i s}$ is bounded, e.g., $\left\|\dddot{\boldsymbol{\tau}}_{d i s}\right\| \leq \delta$ where $\delta>0 \in R$. In other words, any estimation error $(\mathbf{e}(t))$, which lies in a circular plane whose radius is $\left\|\mathbf{e}\left(t_{0}\right)\right\|+\left(\lambda_{\min }(\boldsymbol{\Psi})\right)^{-1} \delta$, converges to a smaller circular plane whose radius is $\left(\lambda_{\min }(\boldsymbol{\Psi})\right)^{-1} \delta$ where $\lambda_{\min }(\boldsymbol{\Psi})$ represents the slowest dynamics of disturbance estimation, i.e., the bandwidth of DOb. The accuracy and convergence rate of disturbance estimation can be simply improved by increasing the bandwidth of DOb; however, it is limited by practical design constraints such as noise and sampling time.

As $\mathbf{e} \rightarrow 0$, the auxiliary variable vectors are accurately estimated, i.e., $\hat{\mathbf{z}}_{1} \rightarrow \mathbf{z}_{1}, \quad \hat{\mathbf{z}}_{2} \rightarrow \mathbf{z}_{2}$ and $\hat{\mathbf{z}}_{3} \rightarrow \mathbf{z}_{3}$. Hence, the estimations of the disturbance vector and its first and second order derivatives are derived as follows:

$$
\begin{aligned}
& \hat{\boldsymbol{\tau}}_{\text {dis }}=\hat{\mathbf{z}}_{1}-L_{1} \mathbf{x} \\
& \hat{\boldsymbol{\tau}}_{\text {dis }}=\hat{\mathbf{z}}_{2}-L_{2} \mathbf{x} \\
& \hat{\boldsymbol{\tau}}_{\text {dis }}=\hat{\mathbf{z}}_{3}-L_{3} \mathbf{x}
\end{aligned}
$$

where $\hat{\boldsymbol{\tau}}_{\text {dis }}, \hat{\boldsymbol{\tau}}_{\text {dis }}$ and $\hat{\vec{\tau}}_{\text {dis }} \in R^{4}$ represent the estimations of $\boldsymbol{\tau}_{\text {dis }}, \dot{\boldsymbol{\tau}}_{\text {dis }}$ and $\ddot{\boldsymbol{\tau}}_{\text {dis }}$, respectively.

\section{Robust TRAJECTORY TRACKING CONTROLLER}

In this section, a new theorem is proposed for a robust trajectory tracking controller that is designed in state space. Reader, who is only interested in the motion control applications of SEAs, can directly follow the next section without going through the theorem.

In order to design a trajectory tracking controller in state space, not only control input but also state references should be adequately generated. One can systematically obtain them in terms of a fictitious design variable, namely differentially flat output, by using DF $[39,40]$.

A linear system is flat, i.e., its state and control input references can be generated in terms of differentially flat output variable, if and only if it is controllable. They are applied to a conventional state feed-back controller so that not only regulation but also trajectory tracking control can be performed [41, 42]. However, a conventional state feed-back controller is sensitive to internal and external disturbances; e.g., assigned poles may significantly change due to plant uncertainties.
Therefore, not only the performance but also the stability may deteriorate in real implementations.

In the following theorem, robust state and control input references are systematically generated by using DF and DOb so that a robust trajectory tracking controller is designed in state space. It has a 2-DOF control structure. Disturbances are cancelled/suppressed by feeding-back their estimations via DOb. Since the system is nominalized by cancelling disturbances, the state feed-back controller can independently adjust the performance in practice.

Theorem: The dynamic model of a linear controllable system can be defined in polynomial matrix form by using

$$
\mathbf{A}_{\mathbf{n}}(s) \mathbf{x}(s)=\mathbf{b}_{\mathbf{n}}(s) u-\boldsymbol{\tau}_{\text {dis }}(s)
$$

where $\mathbf{A}_{\mathbf{n}}(s) \in R^{n \times n}$ represents polynomial nominal system matrix, and it is full rank; $\mathbf{b}_{\mathbf{n}}(s) \in R^{n}$ represents polynomial nominal control input vector; $\mathbf{x}(s) \in R^{n}$ represents states of the system; $u \in R$ represents control input; $\boldsymbol{\tau}_{\text {dis }}(s) \in R^{n}$ represents polynomial disturbance vector; and $s$ represents complex Laplace variable.

The robust state and control input references can be generated by using

$$
\begin{gathered}
\mathbf{x}^{\text {ref }}(s)=\tilde{\mathbf{p}}(s) y_{D F O}=\mathbf{p}_{1}(s) y_{D F O}+\mathbf{P}_{\mathbf{2}}(s) \hat{\boldsymbol{\tau}}_{\text {dis }}^{\text {mismatched }}(s) \\
u^{r e f}=\tilde{q}(s) y_{D F O}=q_{1}(s) y_{D F O}+\mathbf{q}_{\mathbf{2}}^{T}(s) \hat{\boldsymbol{\tau}}_{\text {dis }}^{\text {mathed }}+\mathbf{q}_{\mathbf{3}}^{T}(s) \hat{\boldsymbol{\tau}}_{\text {dis }}^{\text {mismatched }}
\end{gathered}
$$

where $\tilde{\mathbf{p}}(s), \mathbf{p}_{1}(s), \mathbf{q}_{\mathbf{2}}(s)$ and $\mathbf{q}_{\mathbf{3}}(s) \in R^{n} ; \mathbf{P}_{\mathbf{2}}(s) \in R^{n \times n} ; \mathbf{x}^{\text {ref }}$ and $u^{\text {ref }}$ represent state and control input references, respectively; and $\hat{\boldsymbol{\tau}}_{\mathbf{d s}}^{\text {midched }}(s)$ and $\hat{\tau}_{\mathbf{d s}}^{\text {nimudded }}(s) \in R^{u}$ represent the estimations of the matched and mismatched disturbance vectors, respectively.

$\tilde{\mathbf{p}}(s) y_{D F O}$ is derived by solving

$$
\mathbf{c}^{T}(s) \mathbf{A}_{\mathbf{n}}(s) \tilde{\mathbf{p}}(s) y_{D F O}+\mathbf{c}^{T}(s) \hat{\boldsymbol{\tau}}_{\text {dis }}^{\text {mismatched }}(s)=0
$$

where $\mathbf{c}(s) \in R^{n}$ is orthogonal to $\mathbf{b}_{\mathbf{n}}(s)$, i.e., $\mathbf{c}^{T}(s) \mathbf{b}_{\mathbf{n}}(s)=0$.

$$
\begin{aligned}
q_{1}(s), \mathbf{q}_{\mathbf{2}}(s) & \text { and } \mathbf{q}_{\mathbf{3}}(s) \text { are obtained by using } \\
q_{1}(s) & =\left(\mathbf{b}_{\mathbf{n}}{ }^{T}(s) \mathbf{b}_{\mathbf{n}}(s)\right)^{-1} \mathbf{b}_{\mathbf{n}}{ }^{T}(s) \mathbf{A}_{\mathbf{n}}(s) \mathbf{p}_{\mathbf{1}}(s) \\
\mathbf{q}_{\mathbf{2}}(s) & =\left(\mathbf{b}_{\mathbf{n}}{ }^{T}(s) \mathbf{b}_{\mathbf{n}}(s)\right)^{-1} \mathbf{b}_{\mathbf{n}} \\
\mathbf{q}_{\mathbf{3}}(s) & =\left(\mathbf{b}_{\mathbf{n}}{ }^{T}(s) \mathbf{b}_{\mathbf{n}}(s)\right)^{-1} \mathbf{P}_{\mathbf{2}}^{T}(s) \mathbf{A}_{\mathbf{n}}^{T}(s) \mathbf{b}_{\mathbf{n}}(s)
\end{aligned}
$$

Proof: Since the linear system is controllable, states and control input can be defined in terms of differentially flat output variable. Equation (18) can be rewritten as follows:

$$
\begin{aligned}
& \mathbf{A}_{\mathbf{n}}(s) \tilde{\mathbf{p}}(s) y_{D F O}+\mathbf{d}(s) y_{D F O}=\mathbf{b}_{\mathbf{n}}(s) \tilde{q}(s) y_{D F O} \\
& \mathbf{A}_{\mathbf{n}}(s) \tilde{\mathbf{p}}(s)+\mathbf{d}(s)=\mathbf{b}_{\mathbf{n}}(s) \tilde{q}(s)
\end{aligned}
$$

where $\mathbf{x}(s)=\tilde{\mathbf{p}}(s) y_{D F O}, \quad u=\tilde{q}(s) y_{D F O}$, and $\boldsymbol{\tau}_{\text {dis }}(s)=\mathbf{d}(s) y_{D F O}$ in which $\mathbf{d}(s)=\mathbf{b}_{\mathbf{n}}(s) \tilde{q}(s)-\mathbf{A}_{\mathbf{n}}(s) \tilde{\mathbf{p}}(s) \in R^{n}$.

Let us first prove the existence of the solution of Eq. (21). The matched and mismatched disturbances of $\boldsymbol{\tau}_{\text {dis }}(s)$ and $\mathbf{d}(s)$ can be directly separated as follows:

$$
\begin{gathered}
\boldsymbol{\tau}_{\text {dis }}(s)=\boldsymbol{\tau}_{\text {dis }}^{\text {matched }}(s)+\boldsymbol{\tau}_{\text {dis }}^{\text {mismatched }}(s) \\
\mathbf{d}(s)=\mathbf{d}^{\text {matched }}(s)+\mathbf{d}^{\text {mismatched }}(s)
\end{gathered}
$$


If Eq. (24) is multiplied by $\mathbf{c}^{T}(s)$, which is orthogonal to $\mathbf{b}_{\mathbf{h}}(s)$, from left side and Eq. (25) is substituted into the disturbance vector, then Eq. (21) is derived as follows:

$$
\mathbf{c}^{T}(s) \mathbf{A}_{\mathbf{n}}(s) \mathbf{w}(s)=0
$$

where $\mathbf{w}(s)=\tilde{\mathbf{p}}(s)+\mathbf{A}_{\mathbf{n}}^{-1}(s) \mathbf{d}^{\text {mismatched }}(s) \in R^{n}$. Since $\mathbf{A}_{\mathbf{n}}(s)$ is full rank, the polynomial $\tilde{\mathbf{p}}(s)$ can be obtained by using $\tilde{\mathbf{p}}(s)=\mathbf{w}(s)-\mathbf{A}_{\mathbf{n}}^{-1}(s) \mathbf{d}^{\text {mismatched }}(s)$.

Equation (25) shows that the polynomial $\mathbf{w}(s)$ is orthogonal to $\mathbf{A}_{\mathbf{n}}^{T}(s) \mathbf{c}(s)$. It can be obtained by using

$$
\mathbf{w}(s)=\mathbf{R}\left(\mathbf{r}, \frac{\pi}{2}\right) \mathbf{A}_{\mathbf{i}}^{T}(s) \mathbf{c}(s)
$$

where $\mathbf{R}(\mathbf{r}, \theta) \in R^{l \times n}$ is orthogonal rotational matrix; $\mathbf{r} \in R^{n}$ is the axis of rotation; and $\theta \in R$ is the angle of rotation. Equation (27) shows that the polynomial $\tilde{\mathbf{p}}(s)$ has no unique solution.

Equation (22) can be directly derived by multiplying Eq. (23) with $\left(\mathbf{b}_{\mathbf{n}}{ }^{T}(s) \mathbf{b}_{\mathbf{n}}(s)\right)^{-1} \mathbf{b}_{\mathbf{n}}{ }^{T}(s)$ from the left side.

Hence, states and control input are derived in terms of the disturbance vector and differentially flat output variable. The robust state and control input references can be generated by using the estimations of disturbances via $\mathrm{DOb}$ and deriving the differentially flat out variable in terms of control goal.

The block diagram of the proposed robust motion controller is shown in Fig. 2a. Without any simplification, it can be represented by using the analogy of a conventional DOb-based robust motion control system as shown in Fig. 2b. In this figure, $\hat{u}^{\text {rf }}=q_{1}(s) y_{\text {DFO }}$ represents control input reference, $\hat{\mathbf{x}}^{r e f}=\mathbf{p}_{\mathbf{1}}(s) y_{D F O}$ represents state reference, $u^{n}=\hat{u}^{r f f}+\mathbf{K}\left(\hat{\mathbf{x}}^{r f f}-\mathbf{x}\right) \quad$ represents nominal control input signal, $\quad u^{r o b}=\mathbf{q}_{2}^{T}(s) \boldsymbol{\tau}_{\text {dis }}^{\text {mathed }}+$ $\left(\mathbf{q}_{\mathbf{3}}^{T}(s)+\mathbf{K} \mathbf{P}_{\mathbf{2}}(s)\right) \boldsymbol{\tau}_{\text {dis }}^{\text {mismatched }}$ represents robust control input signal, and $\mathbf{K}$ represents state feed-back control gain. It has a 2-DOF control structure. The robustness of the motion control system is achieved by feeding-back the estimations of disturbances in the inner-loop. Since DOb nominalizes the inner-loop by cancelling internal and external disturbances, outer-loop controller, i.e., state feed-back controller, can be designed by only considering the nominal plant model. In order to improve the robustness of the motion control system, the dynamics of disturbance estimation should be faster than that of motion control system. Therefore, the higher the bandwidth of DOb is the more the robustness improves. The stability and performance of the motion control system are simply adjusted by tuning the state feed-back control gain, i.e., $\mathbf{K}$, for nominal system. Reader is invited to refer to [38] for further details of conventional DOb-based 2-DOF motion control systems.

\section{Robust Position AND Force CONTROL of SEAs}

In this section, robust position and force controllers are proposed for SEAs by using DF and DOb. The design philosophy of the robust controllers is explained in the previous

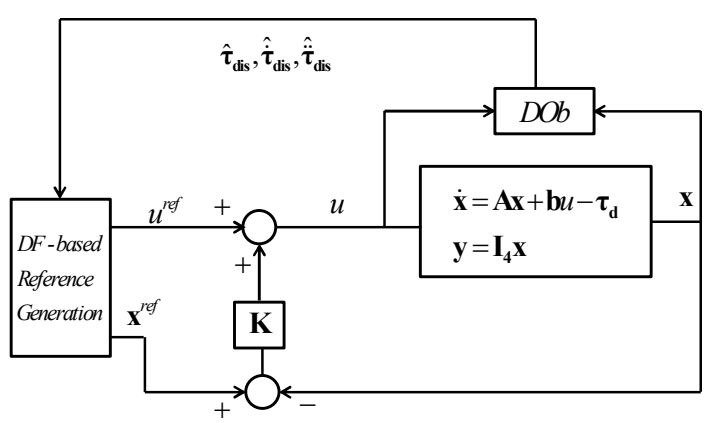

a) Proposed robust motion controller.

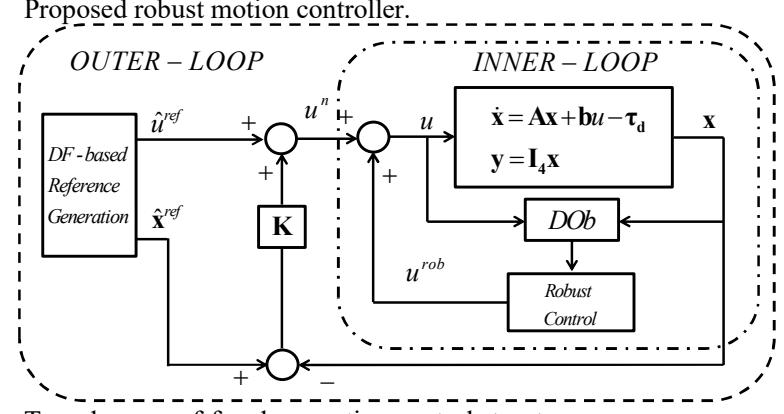

b) Two-degrees-of-freedom motion control structure.

Fig.2: Block diagrams of the proposed robust motion control system.

section. Without going into detail of Theorem, one can directly design the robust motion controllers for SEAs as follows:

Let us first consider the state space dynamic model of an SEA, which is shown in Eq. (4), by neglecting disturbances, i.e., $\boldsymbol{\tau}_{\text {dis }}=\mathbf{0}$. One can easily adjust its nominal stability and performance by using modern control methods such as pole assignment. For example, all nominal poles of an SEA can be placed on the real axis via state feed-back control so that the vibration of the actuator's link is precisely suppressed. If the state feed-back controller, $\mathbf{K}$, is adequately tuned, then all states of the nominal system exponentially go to zero, i.e., regulation is performed. Its state and control input references should be properly generated to perform trajectory tracking control; e.g., they are defined in terms of differentially flat output variable in DF [41]. However, the nominal stability and performance cannot be achieved due to internal and external disturbances of an SEA in practice.

Let us now improve the robustness of an SEA by treating its matched and mismatched disturbances in the design of the controller. The estimations of the disturbances and their first and second order time derivatives are obtained by using the second order DOb in section III. They are fed-back through control input so that disturbances are precisely suppressed. Since an SEA does not suffer from disturbances when ADR control is implemented, its nominal stability and performance can be achieved by using the state feed-back controller in real implementations. The proposed motion controller has 2-DOF; i.e., the robustness and performance can be independently adjusted by using DOb and state feed-back controller, respectively.

The robust state and control input references of the state feedback controller are systematically generated in terms of differentially flat output variable, estimations of disturbances and their successive time derivatives as follows:

The dynamic model of an SEA, which is given in Eq. (1), can be represented in polynomial matrix form by using 


$$
\mathbf{A}_{\mathbf{n}}(s) \mathbf{x}(s)+\boldsymbol{\tau}_{\text {dis }}^{\text {matched }}(s)+\boldsymbol{\tau}_{\text {dis }}^{\text {mismatched }}(s)=\mathbf{b}_{\mathbf{n}}(s) u
$$

where $\mathbf{A}_{\mathbf{n}}(s)=\left[\begin{array}{cc}m_{1 n} s^{2}+b_{1 n} s+k_{12 n} & -k_{12 n} \\ -k_{12 n} & m_{2 n} s^{2}+b_{2 n} s+k_{12 n}\end{array}\right] ; \mathbf{x}(s)=\left[\begin{array}{l}q_{1} \\ q_{2}\end{array}\right]$; $\boldsymbol{\tau}_{\text {dis }}^{\text {mathed }}(s)=\left[\begin{array}{ll}\tau_{d i s}^{\text {matched }} & 0\end{array}\right]^{T} ; \boldsymbol{\tau}_{\text {dis }}^{\text {nismutched }}(s)=\left[\begin{array}{ll}0 & \tau_{d i s}^{\text {nissucher }}\end{array}\right]^{T} ; \quad \mathbf{b}_{\mathbf{n}}(s)=\left[\begin{array}{ll}1 & 0\end{array}\right]^{T} ;$ $u=F_{i n}$; and $s$ represents complex Laplace variable.

Let us multiply Eq. (28) with $\mathbf{c}^{T}(s)=\left[\begin{array}{ll}0 & 1\end{array}\right]$, which is orthogonal to $\mathbf{b}_{\mathbf{n}}(s)$ and $\boldsymbol{\tau}_{\mathbf{d i s}}^{\text {muthed }}(s)$, from the left side.

$$
\mathbf{c}^{T}(s) \mathbf{A}_{\mathbf{n}}(s) \mathbf{x}(s)+\mathbf{c}^{T}(s) \boldsymbol{\tau}_{\text {dis }}^{\text {mismathed }}(s)=0
$$

where $\quad \mathbf{c}^{T}(s) \mathbf{A}_{\mathbf{n}}(s)=\left[\begin{array}{ll}-k_{12 n} & m_{2 n} s^{2}+b_{2 n} s+k_{12 n}\end{array}\right] \quad$ and $\mathbf{c}^{T}(s) \boldsymbol{\tau}_{\text {dis }}^{\text {mismatched }}(s)=\tau_{\text {dis }}^{\text {mismatched }}$.

If it is assumed that $\mathbf{x}(s)=\tilde{\mathbf{p}}(s) y_{D F O}=\left[\begin{array}{ll}\tilde{p}_{1}(s) y_{D F O} & \tilde{p}_{2}(s) y_{D F O}\end{array}\right]^{T}$ where $y_{D F O}$ represents differentially flat output variable, then Eq. (29) is rewritten in terms of $y_{D F O}$ and the mismatched disturbance as follows:

$$
\left[\begin{array}{ll}
-k_{12 n} & m_{2 n} s^{2}+b_{2 n} s+k_{12 n}
\end{array}\right]\left[\begin{array}{l}
\tilde{p}_{1}(s) \\
\tilde{p}_{2}(s)
\end{array}\right] y_{D F O}+\tau_{\text {dis }}^{\text {mismatched }}=0
$$

As shown in Theorem, there is no unique solution for Eq. (30). In this paper, the polynomial vector $\tilde{\mathbf{p}}(s)$ is derived by choosing $\tilde{p}_{2}(s)=k_{12 n}$ as follows:

$\tilde{\mathbf{p}}(s) y_{D F O}=\left[\begin{array}{l}\tilde{p}_{1}(s) \\ \tilde{p}_{2}(s)\end{array}\right] y_{D F O}=\left[\begin{array}{c}\left(m_{2 n} s^{2}+b_{2 n} s+k_{12 n}\right) y_{D F O}+\tau_{d i s}^{n \text { ismatched }} / k_{12 n} \\ k_{12 n} y_{D F O}\end{array}\right]$

where $\tilde{\mathbf{p}}(s) y_{D F O}$ satisfies Eq. (30).

The control input is derived by multiplying Eq. (28) with $\left(\mathbf{b}_{\mathbf{n}}{ }^{T}(s) \mathbf{b}_{\mathbf{n}}(s)\right)^{-1} \mathbf{b}_{\mathbf{n}}{ }^{T}(s)$ from the left side as follows:

$$
\begin{aligned}
& u(s)=\left(m_{1 n} m_{2 n} s^{4}+\left(m_{1 n} b_{2 n}+m_{2 n} b_{1 n}\right) s^{3}+\left(b_{1 n} b_{2 n}+k_{12 n}\left(m_{1 n}+m_{2 n}\right)\right) s^{2}+\right.
\end{aligned}
$$

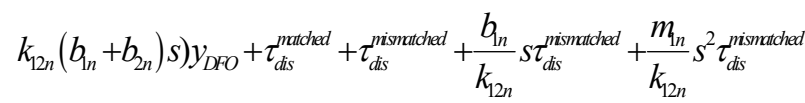

Equation (31) and Eq. (32) show that not only the differentially flat output variable but also disturbances are required to generate state vector and control input. However, disturbances are unknown in many practical motion control applications. In this paper, state vector and control input are generated by using the estimations of disturbances as follows:

$$
\mathbf{x}=\left[\begin{array}{llll}
x_{1} & x_{2} & x_{3} & x_{4}
\end{array}\right]^{T}=\left[\begin{array}{llll}
q_{1} & \dot{q}_{1} & q_{2} & \dot{q}_{2}
\end{array}\right]^{T}
$$

where $x_{1}=m_{2 n} \ddot{y}_{D F O}+b_{2 n} \dot{y}_{D F O}+k_{12 n} y_{D F O}+\hat{\tau}_{d i s}^{\text {mismatched }} / k_{12 n} ; x_{2}=$ $\dot{x}_{1}=m_{2 n} \dddot{y}_{D F O}+b_{2 n} \ddot{y}_{D F O}+k_{12 n} \dot{y}_{D F O}+\hat{i}_{\text {dis }}^{\text {mismatched }} / k_{12 n} ; x_{3}=k_{12 n} y_{D F O}$; $x_{4}=\dot{x}_{3}=k_{12 n} \dot{y}_{D F O}$; and

$$
\begin{aligned}
& u=m_{1 n} m_{2 n} \dddot{\dddot{X}}_{D F O}+\left(m_{1 n} b_{2 n}+m_{2 n} b_{1 n}\right) \dddot{y}_{D F O}+\left(b_{1 n} b_{2 n}+k_{12 n}\left(m_{1 n}+m_{2 n}\right)\right) \ddot{y}_{D F O}+
\end{aligned}
$$

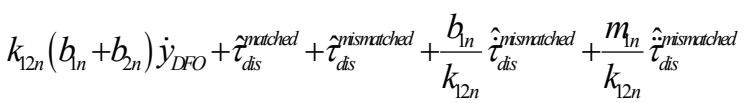

To perform position and force control applications, the desired differentially flat output variable is designed by considering the position and force control goals as follows:

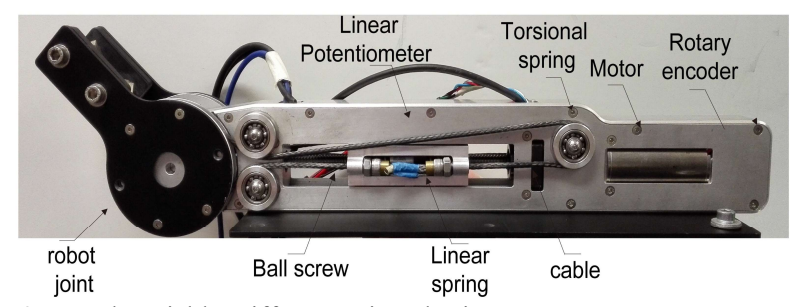

Fig. 3: Novel Variable Stiffness Series Elastic Actuator.

Position Control: The position of the actuator's link is defined in terms of system states and differentially flat output variable by using Eq. (33) as follows:

$$
q_{2}=x_{3}=k_{12 n} y_{D F O}
$$

Let us define the desired position of the actuator's link by using $q_{2}^{\text {des }}$. The desired differentially flat output variable is derived in terms of $q_{2}^{\text {des }}$ by using Eq. (35) as follows:

$$
y_{D F O}^{d e s}=\frac{x_{3}^{d e s}}{k_{12 n}}=\frac{q_{2}^{d e s}}{k_{12 n}}
$$

where $\bullet^{d e s}$ represents desired $\bullet$. The robust state and control input references of the position controller are generated by applying Eq. (36), i.e., $y_{D F O}=y_{D F O}^{\text {des }}$, into Eq. (33) and Eq. (34).

Force Control: Similarly, spring force is defined in terms of system states and differentially flat output variable by using Eq. (33) as follows:

$$
F_{\text {spring }}=k_{12 n}\left(x_{1}-x_{3}\right)=k_{12 n}\left(m_{2 n} \ddot{y}_{D F O}+b_{2 n} \dot{y}_{D F O}\right)+\hat{\tau}_{\text {dis }}^{\text {nismatched }}
$$

Let us define the desired spring force by using $F_{\text {sping }}^{d s}$. The desired differentially flat output variable is derived in terms of $F_{\text {sping }}^{d k s}$ by using Eq. (37) as follows:

$$
\ddot{y}_{D F O}^{\text {des }}=\frac{F_{\text {spring }}^{\text {des }}-\hat{\tau}_{d i s}^{\text {nismatched }}-b_{2 n} k_{12 n} \dot{y}_{D F O}^{d e s}}{k_{12 n} m_{2 n}}
$$

The robust state and control input references of the force controller are similarly generated by applying Eq. (38) into Eq. (33) and Eq. (34).

Hence, either position or force control applications of an SEA can be performed by using the proposed unified ADR motion controller.

\section{EXPERIMENTS}

In this section, robust position and force control experimental results of an SEA, which is shown in Fig. 3, are given to validate the proposed controllers. It has a novel mechanical structure which consists of torsional and linear springs in series. By adjusting the compliance of the springs, e.g. hard torsional and soft linear springs, a compact variable-stiffness SEA can be designed. The reader is invited to refer to $[5,17]$ for further details on the novel mechanical design of the two-state variable stiffness SEA. However, in this paper, only the torsional spring, i.e., a conventional SEA is used to validate our proposals. The specifications of the experimental setup are given in Table I. The center processor is dSPACE DS1007 with DS3002 counter board to collect encoder signals and DS2102 DAC board to control motor driver. The experiments were performed by using $2 \mathrm{KHz}$ sampling frequency. The resolutions of the encoders are 2048 and 1024 at motor and link sides, respectively. 
TABLE I

SPECIFICATIONS OF EXPERIMENTAL SETUP

\begin{tabular}{ccc}
\hline \hline Parameter & \multicolumn{1}{c}{ Value } & Description \\
\hline $\mathrm{m}_{1 \mathrm{n}}$ & $2.2 \times 10^{-6} \mathrm{Kgm}^{2}$ & Nominal mass \\
$\mathrm{m}_{2 \mathrm{n}}$ & $4 \times 10^{-6} \mathrm{Kgm}^{2}$ & Nominal mass \\
$\mathrm{k}_{12 \mathrm{n}}$ & $0.14 \mathrm{Nm} / \mathrm{rad}$ & Stiffness of the spring \\
\hline \hline
\end{tabular}

Section VI is divided into two subsections, namely position control and force control. In the former, the control goal is defined as the desired position of the actuator's link. However, in the latter, the control goal is defined as the desired deflection of the actuator's spring. It is widely used in the force control applications of SEAs. In order to evaluate the position and force control performances, step and sinusoidal reference inputs are applied in regulation and trajectory tracking control applications, respectively. It is experimentally shown that

- The link of the actuator can precisely follow step and sinusoidal reference inputs by suppressing internal and external disturbances when the proposed robust position controller is implemented.

- The spring force, i.e., the deflection of the spring, can precisely follow step and sinusoidal reference inputs when the proposed robust force controller is implemented.

The robust position and force controllers are designed by using the following three steps:

Step1. The state feed-back controller is designed for the nominal plant model by neglecting internal and external disturbances, i.e., $\boldsymbol{\tau}_{\mathbf{d s}}=\mathbf{0}$. For example, $\mathbf{K}=\operatorname{acker}\left(\mathbf{A}_{\mathbf{n}}, \mathbf{b}_{\mathbf{n}}, \mathbf{p}^{\text {des }}\right)$, in which $\mathbf{p}^{\text {des }}$ represents the desired poles, command of MATLAB can be used to automatically obtain the sate feed-back controller gain. The desired poles are experimentally tuned; e.g., as they are increased, the motion control system becomes more noise-sensitive; however, as they are decreased, the performance deteriorates.

Step2. The robust state and control input references are generated by using Eq. (33) and Eq. (34), respectively.

Step3. To perform the robust position and force control applications by using the unified motion controller, the differentially flat output variable is derived as follows:

3.1. Equation (36) is used so that robust position control is performed.

3.2. Equation (38) is used so that robust force control is performed.

Note that the conventional DF-based position controller is designed by neglecting the estimations of disturbances and their successive derivatives in Eq. (33) and Eq. (34).

\section{A. Position Control:}

Let us first consider point-to-point position control, i.e., position regulation, problem of the actuator's link. By using Step1, the state feed-back controller is tuned as $\mathbf{K}=\left[\begin{array}{llll}-0.1056 & 0.0007 & 0.1119 & -0.0006\end{array}\right]$ so that all poles of the nominal system are placed at -100 . The robust state and control input references are generated by using Step2, and the desired differentially flat output variable of position control is derived by using Step3.1. A step reference input, which has $0.32 \mathrm{rad}$ amplitude, is applied after 5 seconds. To evaluate the robustness of the position controllers, the actuator is disturbed by directly

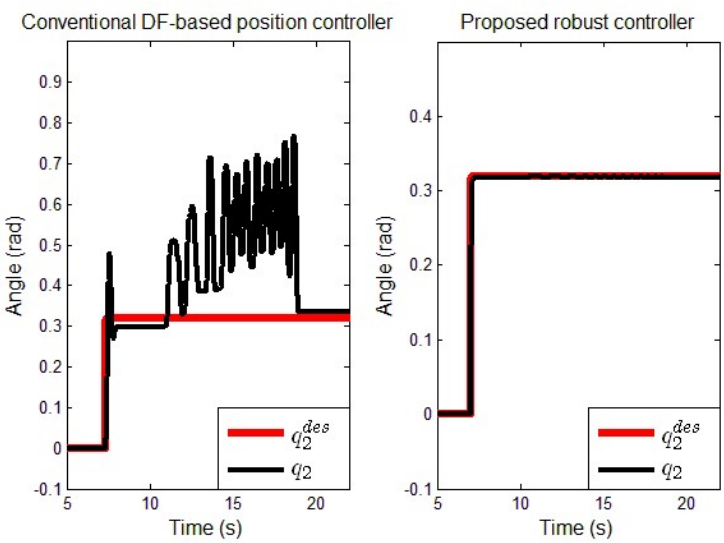

a)
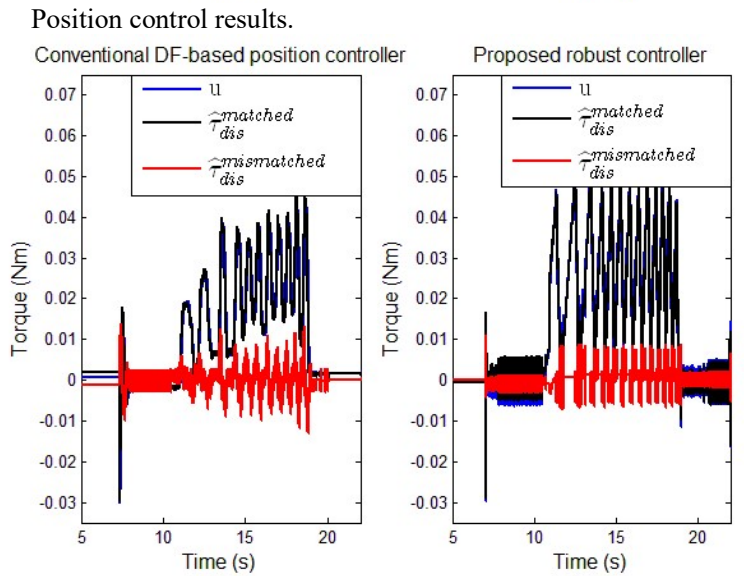

b) Estimations of disturbances and control inputs.

Fig. 4: Position regulation control results when the proposed robust and conventional DF-based position controllers are implemented. The bandwidth of $\mathrm{DOb}$ is $700 \mathrm{rad} / \mathrm{s}$.

pulling and pushing its link with hand; i.e., a variable unknown and dynamic external disturbance is applied. Figure 4a shows the position control results when the conventional DF-based position controller and the proposed robust position controller are implemented. The former is sensitive to internal and external disturbances. It suffers from steady state error between 8 to 11 and 19 to 22 seconds due to internal disturbances such as friction. Moreover, its performance significantly deteriorates when the external disturbance is applied between 11 and 19 seconds. The proposed robust controller improves the performance of the position control of SEA by suppressing disturbances. The link of the actuator can precisely follow step reference input when the SEA suffers from internal and external disturbances. Figure $4 \mathrm{~b}$ illustrates the estimations of matched and mismatched disturbances. It is clear from the figure that similar external disturbances are applied when the proposed robust and conventional DF-based position controllers are implemented. Control signals of the position control experiments are also illustrated in this figure. The robustness of the position control is improved without requiring high control signals thanks to the proposed ADR control structure in which disturbances are cancelled by feeding-back their estimations.

To evaluate the robustness and noise-sensitivity of the proposed position controller, let us re-perform the regulation experiment by using different bandwidth values of DOb. In this experiment, the state-feedback controller is tuned as

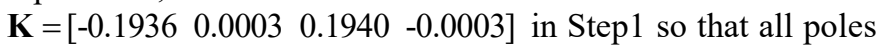
of the nominal system are placed at -50 . It is more sensitive to 

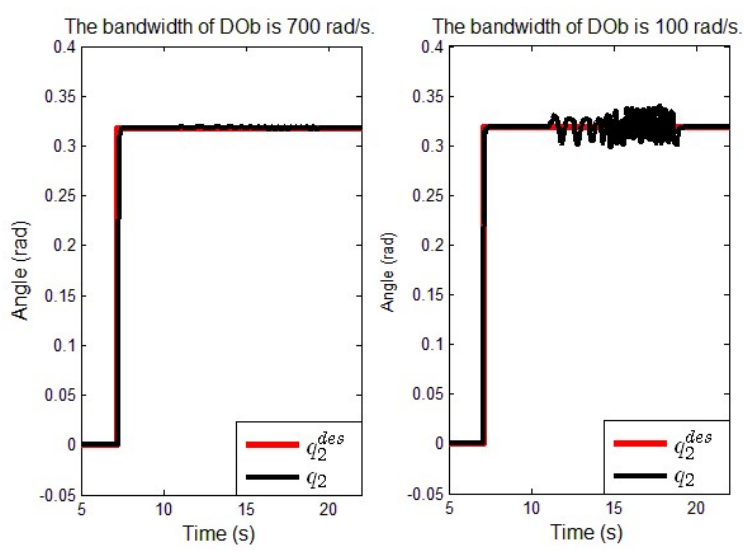

a) Robust position control results.
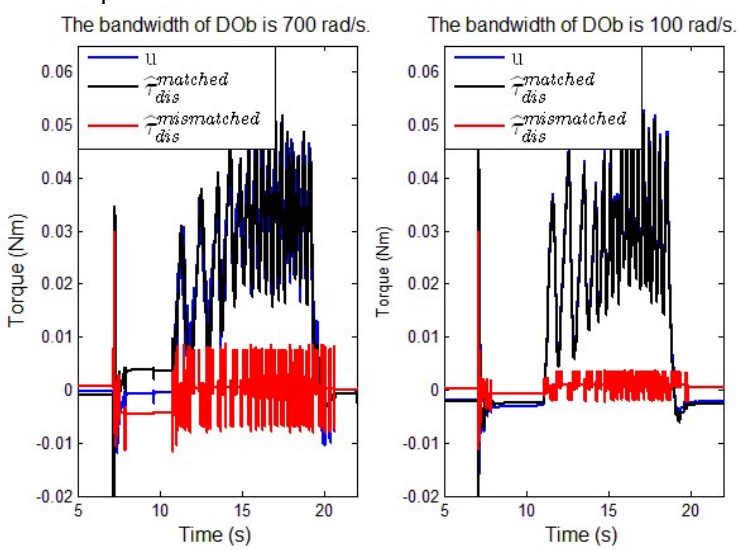

b) Estimations of disturbances and control inputs.
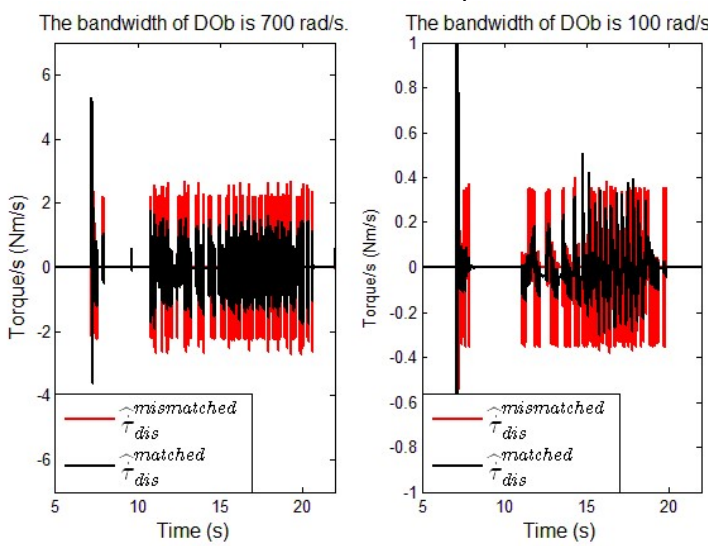

c) Estimations of the first order derivatives of disturbances.

Fig. 5: Position regulation control results when different bandwidth values are used in the design of $\mathrm{DOb}$.

disturbances than the previous state feed-back controller. The position controller is similarly designed by following Step2 and Step3.1. The link of the actuator follows the same reference input and suffers from similar external disturbances. Figure 5a shows the position control results when different bandwidth values of $\mathrm{DOb}$ are used in the design of the proposed robust position controller. It is clear from the figure that the higher the bandwidth of $\mathrm{DOb}$ is the more the robustness improves. To achieve high performance, i.e., precisely suppress disturbances, the bandwidth of DOb should be increased. Control signals of the robust position control experiments are illustrated in Fig. $5 \mathrm{~b}$. It shows that as the bandwidth of DOb is increased, the control signal does not dramatically change; however, it includes more noise. The estimations of disturbances and their first derivatives are illustrated in Fig. 5b and Fig. 5c, respectively. The noise of disturbance estimation increases when DOb is designed by using higher bandwidth values. It is the main source of the control signal noise in Fig. 5b. The experiment clearly shows that there is a trade-off between the robustness and noise-sensitivity in the design of the proposed position controller.

Let us now consider the trajectory tracking control problem of the actuator's link. The state feed-back controller is designed

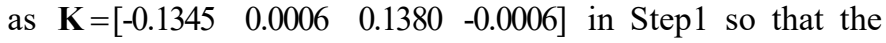
double poles of the nominal system are placed at -75 and -100 . The trajectory tracking controller is similarly designed by following Step2 and Step3.1. The bandwidth of DOb is set as

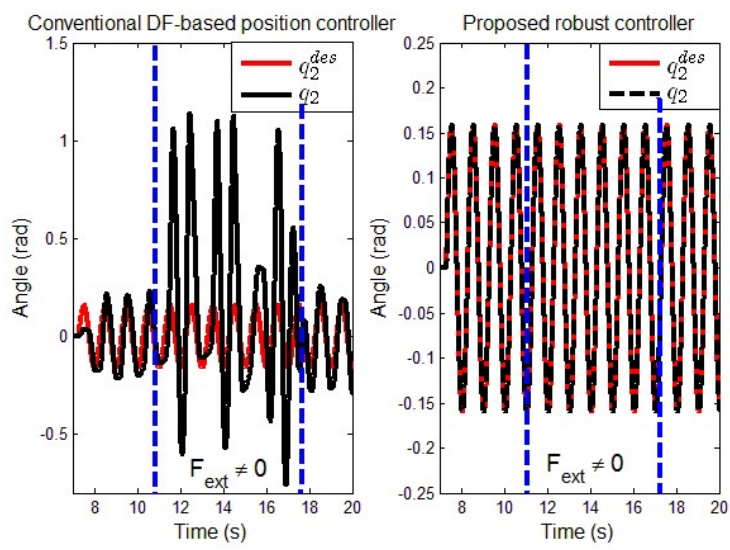

a) Sinusoidal trajectory tracking control results when $\mathrm{f}=1 \mathrm{~Hz}$.
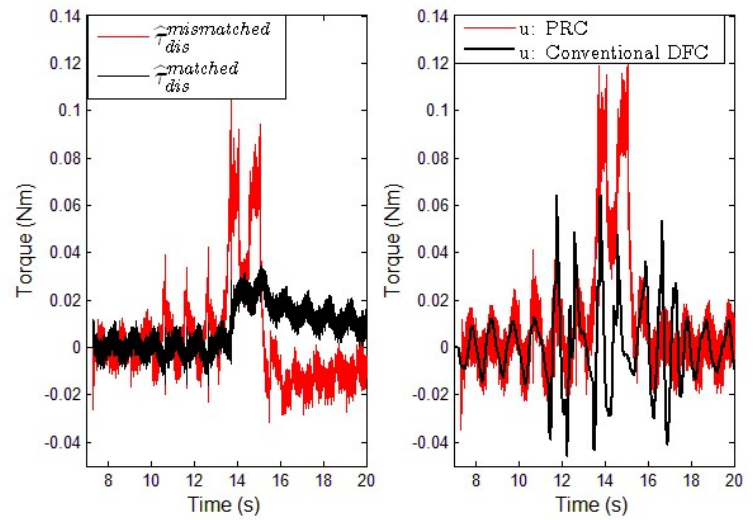

b) Estimations of disturbances and control inputs. PRC: Proposed robust controller; DFC: Differential Flatness-based Controller

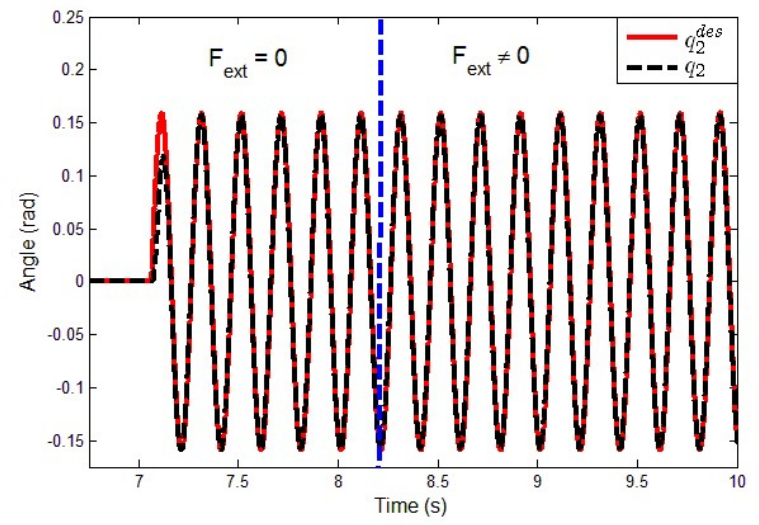

c) Sinusoidal trajectory tracking control results when $\mathrm{f}=5 \mathrm{~Hz}$.

Fig. 6: Position trajectory tracking control results when the proposed robust and conventional DF-based position controllers are implemented. 
$750 \mathrm{rad} / \mathrm{s}$. Sinusoidal reference inputs, which have $0.16 \mathrm{rad}$ amplitude and $1 \mathrm{~Hz}$ and $5 \mathrm{~Hz}$ frequencies, are applied after 7 seconds. Two different external disturbances are applied as follows: a constant known external disturbance is applied by hanging a 2-kg weight to the link of the actuator; a variable unknown and dynamic external disturbance is applied by directly pulling and pushing the link of the actuator with hand. Figure 6a shows the trajectory tracking control results when the proposed robust and conventional DF-based position controllers are implemented. The former improves the performance of trajectory tracking control by suppressing disturbances. The link of the actuator can accurately follow sinusoidal reference input. However, the conventional controller suffers from not only external but also internal disturbances. The link of the actuator cannot precisely follow the desired trajectory due to internal disturbances between 7 to
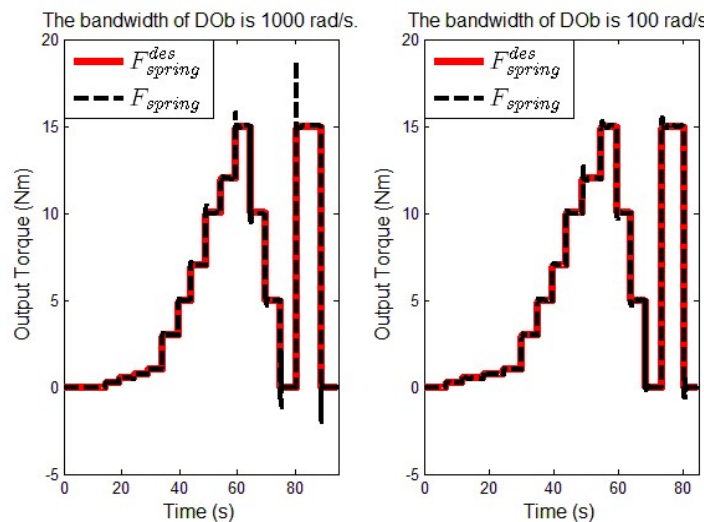

a)

Robust force control results. Output torque.

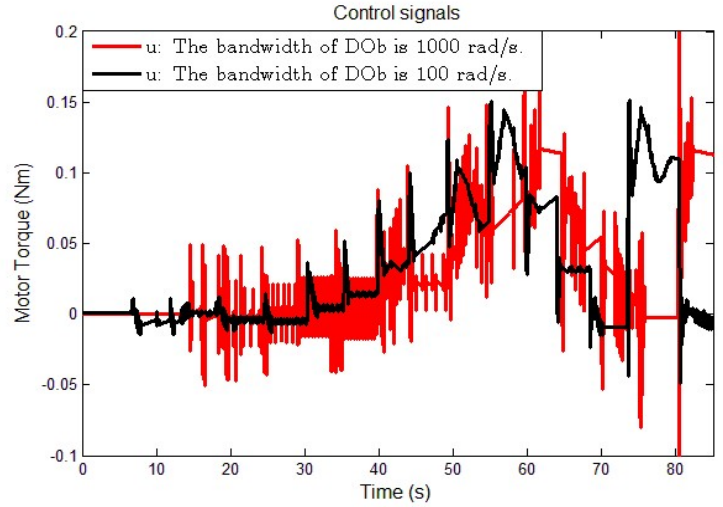

b) Control signals. Motor torque.

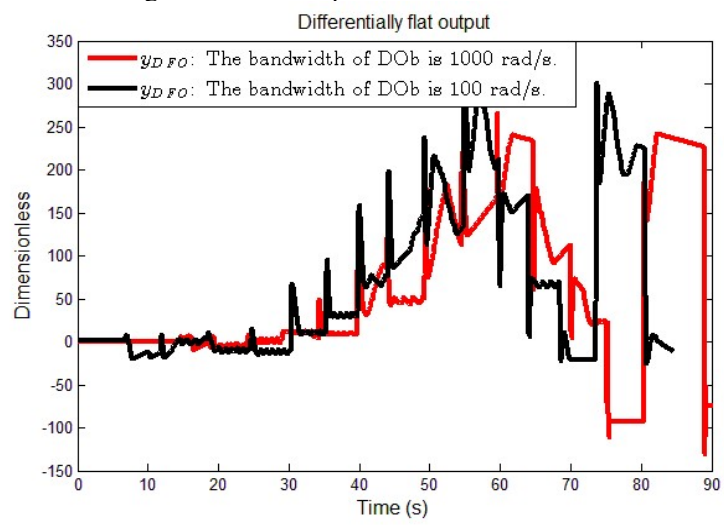

c) Differentially flat output variable.

Fig. 7: Robust force regulation control results when different bandwidth values are used in the design of DOb.
11 and 18 to 20 seconds. Plus, its performance significantly deteriorates when external disturbances are applied between 11 and 18 seconds. Figure $6 \mathrm{~b}$ illustrates the estimations of disturbances and control signals. The proposed robust position controller automatically adjusts the control signal and suppresses disturbances when they are applied. Thanks to the proposed ADR control structure, the robustness of the position control is obtained without using high control signals. Figure 6c shows that high frequency reference trajectories can be precisely followed by using the proposed robust position controller.

\section{B. Force Control:}

Let us first consider the force regulation control problem of SEA. In order to assign the double poles of the nominal system at -75 and -100 , the state feed-back controller is similarly

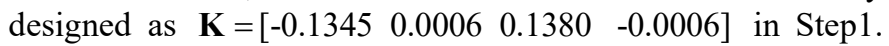

The robust state and control input references are similarly generated by using Step2; however, the desired differentially flat output variable of force control is derived by using Step3.2. Several step reference inputs are consecutively applied by using $0.25 \mathrm{Nm}, 0.5 \mathrm{Nm}, 0.75 \mathrm{Nm}, 1 \mathrm{Nm}, 3 \mathrm{Nm}, 5 \mathrm{Nm}, 7 \mathrm{Nm}, 10 \mathrm{Nm}$, $13 \mathrm{Nm}, 15 \mathrm{Nm}, 10 \mathrm{Nm}, 5 \mathrm{Nm}, 0 \mathrm{Nm}, 15 \mathrm{Nm}$ and $0 \mathrm{Nm}$. A sponge is placed between the link of the actuator and a metal environment so that the stability and performance of contact motion are evaluated for different environmental dynamics, i.e., stiffness. As the force control input is increased, the dynamics of the rigid environment (metal) becomes more dominant. Figure 7a illustrates the force regulation control results when the proposed robust force controller is implemented by using different bandwidth values of DOb. At low force reference range, the actuator contacts to soft environment (sponge); however, as the force reference input is increased, it starts to contact to hard environment (metal). The figure clearly shows that stable contact motion can be achieved for different environmental dynamics, and the force regulation control can be precisely performed for different bandwidth values of DOb. Control input signals of robust force control experiments are illustrated in Fig. 7b. Similar to the robust position control experiments, as the bandwidth of DOb is increased, the robust force control system becomes more noise-sensitive. However, high performance force regulation control experiments can be performed for low bandwidth values of DOb. Figure 7c illustrates the fictitious differentially flat output variable of the robust force regulation control experiment.

Lastly, let us consider the force trajectory tracking control problem of SEA. The state feed-back controller is designed as $\mathbf{K}=\left[\begin{array}{llll}-0.0445 & 0.0009 & 0.0587 & -0.0006\end{array}\right]$ so that the double poles of the nominal system are placed at -120 and -125 in Step1. The robust force controller is similarly designed by following Step2 and Step3.2. Trajectory reference inputs are applied by using $F_{\text {spring }}^{\text {des }}=7+3 \sin \left(2 \pi f_{i} t\right) \mathrm{Nm}$ where $i=1,2$, and $f_{l}=1 \mathrm{~Hz}$ and $f_{2}$ $=1 \mathrm{~Hz}$. The link of the actuator initially contacts to a rigid environment, i.e., metal. Figure 8a shows force trajectory tracking control results when the frequency of sinusoidal reference input is $1 \mathrm{~Hz}$ and the robust force controller is designed by using different bandwidth values of DOb. Force trajectory tracking control can be precisely performed when the proposed robust force controller is implemented. Its 

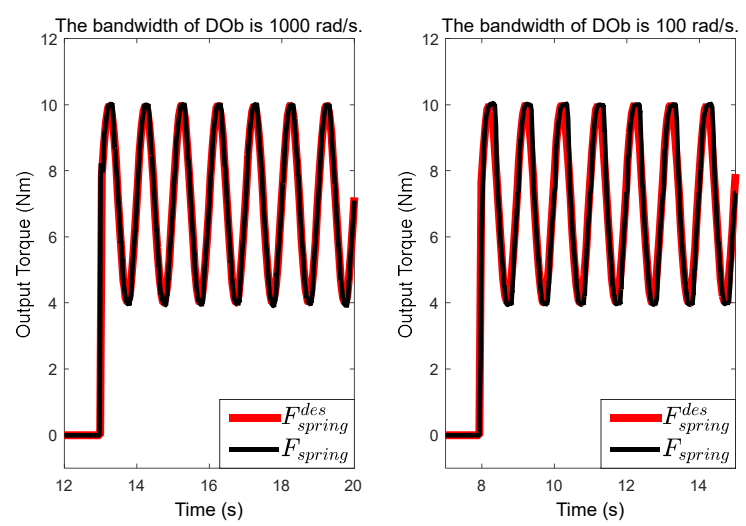

a) Robust force control results. Output torque $\mathrm{f}=1 \mathrm{~Hz}$.

Control signals

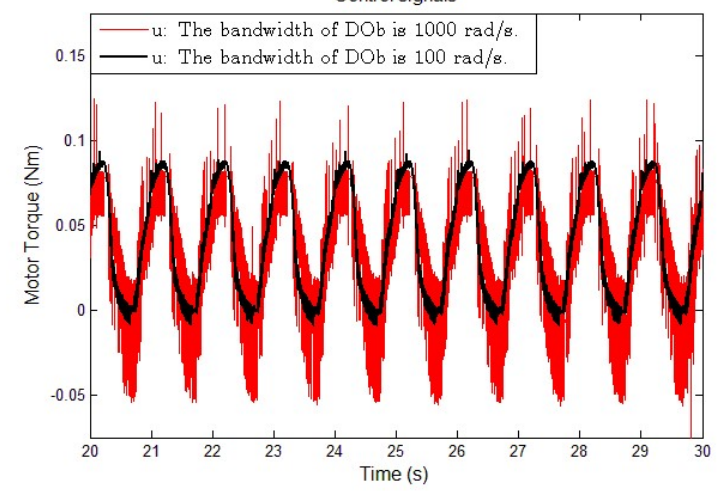

b) Control input signals. $\mathrm{f}=1 \mathrm{~Hz}$.

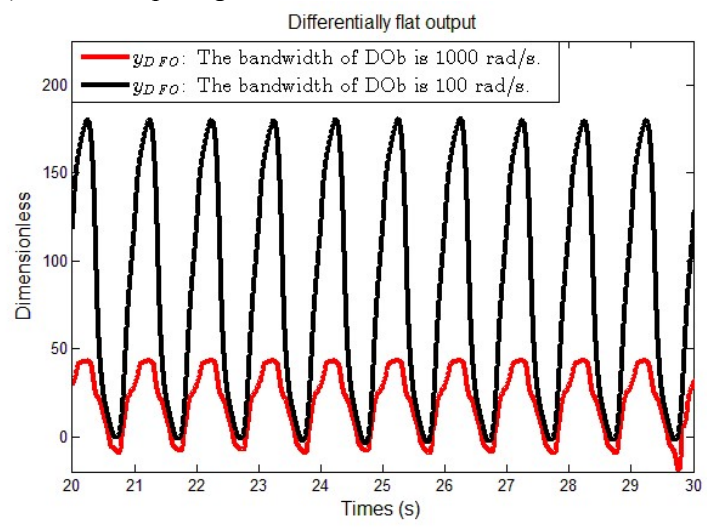

c) Differentially flat output variable.

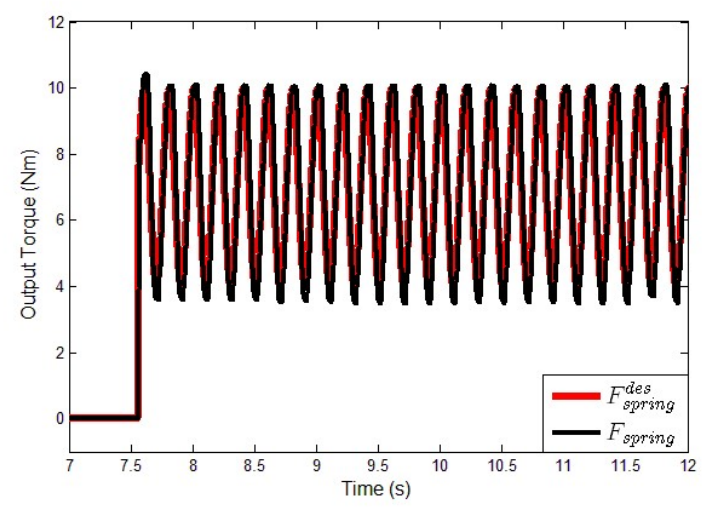

d) Robust force control result. Output torque. $\mathrm{f}=5 \mathrm{~Hz}$.

Fig. 8: Robust force trajectory tracking control results when different bandwidth values are used in the design of DOb.

performance is improved as the bandwidth of DOb is increased. However, as shown in Fig. 8b, it is limited by noise in practice.
The trade-off between the robustness and noise sensitivity should be considered in the design of the robust force controller as well. Figure $8 \mathrm{c}$ shows the differentially flat output variable when the frequency of the force reference input is $1 \mathrm{~Hz}$. The fluctuation of differentially flat output variable is suppressed by using better disturbance estimation. Figure $8 \mathrm{~d}$ shows the robust force trajectory tracking control result when the frequency of sinusoidal reference input is $5 \mathrm{~Hz}$. It is clear from the figure that high frequency trajectory reference can be precisely tracked when the proposed robust force controller is implemented.

The position and force control performances of an SEA are limited when conventional PID controllers are implemented as shown in [17]. In this section, similar position control performance limitations are shown when conventional DFbased controller is implemented. It is experimentally shown that the proposed robust controller significantly improves the position and force control performances of SEAs by suppressing internal and external disturbances. Against resonance ratio controller [17], it can suppress not only constant but also variable disturbances. Therefore, it is very practical for motion control applications. The robustness of the proposed motion controller can be simply improved by increasing the bandwidth of DOb. However, it is limited by practical design constraints such as noise and sampling time. They are directly related to experimental setup; e.g., noise can be suppressed by using high resolution encoders in practice. However, it increases cost. The trade-off between the robustness and noisesensitivity should be considered in the design of the proposed robust motion controller.

\section{CONCLUSION}

This paper has proposed a unified ADR motion controller for the robust position and force control problems of SEAs in state space. It is designed by combining DF and DOb so that not only the state and control input references of the state feed-back controller are generated but also its robustness is achieved. The proposed motion controller has 2-DOF; its performance and robustness can be adjusted by separately tuning the state feedback controller and $\mathrm{DOb}$, respectively. The former, i.e., performance controller, is designed by neglecting internal and external disturbances; e.g., the vibration of the actuator's link is suppressed by assigning all poles of the nominal system on the real axis. The robustness is achieved by feeding-back the estimations of disturbances thanks to the proposed ADR control structure. The robust state and control input references of the state feed-back controller are systematically generated in terms of the differentially flat output variable, the estimations of disturbances and their successive derivatives in Theorem. They are estimated by designing a second order $\mathrm{DOb}$ in state space. To improve the robustness of the proposed controller, i.e., the performance of disturbance estimation, the poles of DOb should be faster than that of the nominal system. Faster poles in the design of DOb correspond to its higher bandwidth. However, it is limited by practical design constraints, such as noise and sampling time, and cannot be freely increased in real implementations. The trade-off between the robustness and noise sensitivity is clarified by giving experimental results in section VI. It is shown that the proposed robust motion controller significantly improves the position and force control 
performances of an SEA without requiring its precise dynamic model. It is validated by giving position and force control experimental results of an SEA.

\section{REFERENCES}

[1] G. Zeng, "An overview of robot force control," Robotica, vol. 15, no. 15, pp. 473-482, Sep. 1997.

[2] M. Grotjahn, B. Heimann, "Model-based feedforward control in industrial robotics," Int. J. Robot Res., vol. 21, no. 1, pp. 45-60, Jan. 2002.

[3] Y. M. Zhao, Y. Lin, F. Xi, S. Guo, "Calibration-Based Iterative Learning Control for Path Tracking of Industrial Robots," IEEE Trans. Ind. Electron., vol.62, no.5, pp.2921-2929, May 2015.

[4] G. Pratt, M. Williamson, "Series elastic actuators," in Proc. IEEE/RSJIROS, Pittsburgh, PA, USA, 1995, pp. 399-406.

[5] H. Yu, S. Huang, G. Chen, Y. Pan, Z. Guo, "Human-Robot Interaction Control of Rehabilitation Robots with Series Elastic Actuators," IEEE Trans. Robot., vol. 31, no. 99, pp. 1-12, Oct. 2015.

[6] C- L Fok, G. Johnson, J. D. Yamokoski, A. Mok, L. Sentis. "ControlIt! A Middleware for Whole-Body Operational Space Control," Int. J. Human. Robot., vol. 13, no. 1, pp.1-57 (1550040), Mar. 2016.

[7] N.M. Ceriani, A. M. Zanchettin, P. Rocco, A. Stolt, A. Robertsson, "Reactive Task Adaptation Based on Hierarchical Constraints Classification for Safe Industrial Robots," IEEE/ASME Trans. Mechatronics, vol.20, no.6, pp. 2935-2949, Dec. 2015.

[8] N. Hogan, "Impedance Control: An Approach to Implementation," part IIII, J. Dyn. Syst. Meas. Control., vol. 107, no. 1, pp. 1-24, Mar. 1985.

[9] T.Lefebvre, J. Xiao, H. Bruyninckx, G. de Gersem, "Active compliant motion: a survey," Adv. Robot., vol. 19, issue 5, pp. 479-499, 2005.

[10] E. Sariyildiz, K. Ohnishi, "An Adaptive Reaction Force Observer Design," IEEE/ASME Trans. Mechatronics, vol. 20, no. 2, pp. 750-760, Apr. 2015.

[11] E. Sariyildiz, K. Ohnishi, "On the Explicit Robust Force Control via Disturbance Observer", IEEE Trans. Ind. Electron., vol. 62, no. 3, pp. 1581-1589, Mar. 2015.

[12] T.S. Tadele, T. de Vries, S. Stramigioli, "The Safety of Domestic Robotics: A Survey of Various Safety-Related Publications," IEEE Robot. Autom. Mag., vol.21, no.3, pp.134-142, Sept. 2014.

[13] A. G. Buizzai, N. M. Ceriani, A. M. Zanchettin, P. Rocco, L. Bascetta, "Safety Control of Industrial Robots Based on a Distributed Distance Sensor," IEEE Trans. on Control Syst. Technol., vol.22, no.6, pp.21272140, Nov. 2014

[14] J. Hurst, J. Chestnutt, A. Rizzi, "The actuator with mechanically adjustable series compliance," IEEE Trans. Robot., vol. 26, no 4, pp. 597606, Aug. 2010.

[15] B. Vanderborght et. al., "Variable impedance actuators: A review," Robot. Auton. Syst., vol. 61, pp. 1601-1614, Dec. 2013.

[16] D.W. Robinson, J.E. Pratt, D.J. Paluska, G.A. Pratt, "Series elastic actuator development for a biomimetic walking robot," in Proc. IEEE/ASME-AIM, Atlanta, GA, USA, 1999, pp.561-568.

[17] E. Sariyildiz, G. Chen, H. Yu, "An Acceleration-Based Robust Motion Controller Design for a Novel Series Elastic Actuator," IEEE Trans. Ind. Electron., vol. 63, no. 3, pp. 1900-1910, Mar. 2016.

[18] L. Jinoh, H. Dallali, N. Tsagarakis, D. Caldwell, "Robust and model-free link position tracking control for humanoid COMAN with multiple compliant joints," IEEE-RAS Humanoids, Atlanta, USA, 2013, pp.

[19] M. W. Spong, K. Khorasani, P. V. Kokotovic, "An integral manifold approach to the feedback control of flexible joint robots," IEEE J. Robot. Autom., vol.3, no.4, pp.291-300, Aug. 1987.

[20] G.A. Wilson, G.W. Irwin, "Robust tracking of elastic joint manipulators using sliding mode control," Trans. Inst. Measurement Control, vol. 16, no. 2, pp. 99-107, Apr. 1994.

[21] Z. Chen, B. Yao, Q. Wang, "mu-synthesis-based adaptive robust control of linear motor driven stages with high-frequency dynamics: A Case Study," IEEE/ASME Trans. Mechatronics, vol. 20, no. 3, pp. 1482-1490, June 2015.

[22] P. Tomei, "A simple PD controller for robots with elastic joints", IEEE Trans. Autom. Control, vol.36, no.10, pp.1208-1213, Oct. 1991.

[23] A. De Luca, F. Flacco, "A PD-type regulator with exact gravity cancellation for robots with flexible joints," IEEE-ICRA, Shanghai, China, 2011, pp. 317-323.

[24] A. Albu-Schaffer, G. Hirzinger, "State feedback controller for flexible joint robots: a globally stable approach implemented on DLR's light- weight robots," in Proc. IEEE/RSJ-IROS, Takamatsu, Japan, 2000, pp. 1087-1093.

[25] I. N. Kar, K. Seto, and F. Doi, "Multimode vibration control of a flexible structure using Ho-based robust control," IEEE/ASME Trans. Mechatronics, vol. 5, no. 1, pp. 23-31, Mar. 2000.

[26] H. N. Rahimi, M. Nazemizadeh, "Dynamic analysis and intelligent control techniques for flexible manipulators: a review," $A d v$. Robot., vol. 28, no. 2, pp. 63-76, 2014.

[27] V. Zeman, R. V. Patel, K. Khorasani, "Control of a flexible-joint robot using neural networks", IEEE Trans. Control Syst. Technol., vol.5, no.4, pp.453-462, Jul 1997.

[28] E. Sariyildiz, K. Ohnishi, "A Guide to Design Disturbance Observer," $J$. Dyn. Syst. Meas. Control, vol. 136, no. 2, pp. 1-10 (021011), Mar. 2014.

[29] G. A. Pratt, P. Willisson, C. Bolton, A. Hofman, "Late motor processing in low-impedance robots: Impedance control of series-elastic actuators," in Proc. IEEE-ACC, Boston, MA, USA, 2004, pp. 3245-3251.

[30] H. Vallery, J. Veneman, E. van Asseldonk, R. Ekkelenkamp, M. Buss, H. van Der Kooij, "Compliant actuation of rehabilitation robots," IEEE Robot. Autom. Mag., vol.15, no.3, pp.60-69, Sept. 2008.

[31] K. Kong, J. Bae, and M. Tomizuka, "Control of rotary series elastic actuator for ideal force-mode actuation in human-robot interaction applications," IEEE/ASME Trans. Mechatronics, vol. 14, pp. 105-118, Feb. 2009

[32] N. Paine, S. Oh, L. Sentis, "Design and Control Considerations for HighPerformance Series Elastic Actuators," IEEE/ASME Trans. Mechatronics, vol. 19, no. 3, pp. 1080-1091, June 2014.

[33] N. Paine, J. S. Mehling, J. Holley, N. Radford, G. Johnson, C-L Fok, L. Sentis, "Actuator control for the NASA-JSC Valkyrie humanoid robot: A decoupled dynamics approach for torque control of series elastic robots," J. Field Robot., vol. 32, no. 3, pp. 378-396, May 2015.

[34] J. Bae, K. Kong, M. Tomizuka, "Gait phase-based smoothed sliding mode control for a rotary series elastic actuator installed on the knee joint," in Proc. IEEE-ACC, Baltimore, MD, USA, 2010, pp. 6030-6035.

[35] S.H. Li, J. Yang, W.H. Chen, and X.S. Chen, "Generalized extended state observer based control for systems with mismatched uncertainties," IEEE Trans. Ind. Electron., vol. 59, no. 12, pp. 4792-4802, Dec. 2012.

[36] S. Li, H, Sun, J, Yang, X, and Yu, "Continuous Finite-Time Output Regulation for Disturbed Systems Under Mismatching Condition," IEEE Trans. on Autom. Control, vol.60, no.1, pp. 277-282, Jan. 2015.

[37] X. J. Wei and L. Guo, "Composite disturbance-observer-based control and H-infinity control for complex continuous models," Int. J. Robust Nonlin, vol. 20, no. 1, pp. 106-118, Jan. 2010.

[38] E. Sariyildiz, K. Ohnishi, "Stability and Robustness of Disturbance Observer Based Motion Control Systems," IEEE Trans. Ind. Electron., vol. 62, no. 1, pp. 414-422, Jan. 2015.

[39] M. Fliess, J. Levine, Ph. Martin, P. Rouchon, "Flatness and defect of nonlinear systems: introductory theory and applications", Int. J. Control, vol. 61, pp. $1327-1361,1995$.

[40] C. H. Ko, K. Y. Young, Y. C. Huang, S. K. Agrawal, "Walk-Assist Robot: A novel approach to gain selection of a braking controller using differential flatness," IEEE Trans. Control Syst. Technol., vol. 21, no. 6, pp. 2299-2305, Nov. 2013.

[41] J. Levine and D.V. Nguyen, "Flat output characterization for linear systems using polynomial matrices," Syst. Control Lett., vol. 48, pp. $69-$ 75, Jan. 2003

[42] S. Z. Yong, B. Paden, E. Frazzoli, "Computational methods for MIMO flat linear systems: Flat output characterization, test and tracking control," in Proc. IEEE-ACC, Chicago, IL, USA, 2015, pp. 3898-3904.

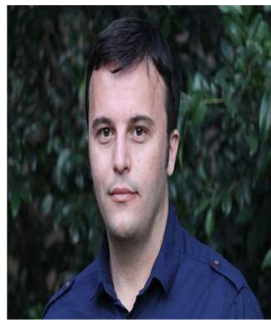

Emre Sariyildiz (S'11, M'16) received his first Ph.D. degree in Integrated Design Engineering from Keio University, Tokyo, Japan, in September 2014 and second Ph.D. degree in Control and Automation Engineering from Istanbul Technical University, Istanbul, Turkey, in February 2016. He was a research fellow in the department of Biomedical Engineering and Singapore Institute for Neurotechnology (SINAPSE) at National University of Singapore, Singapore, between 2014 and 2017. Since April 2017, he has been Lecturer in the School of 
Mechanical, Materials, Mechatronic and Biomedical Engineering at University of Wollongong, NSW, Australia. His main research interests are control theory, robotics, mechatronics and motion control.

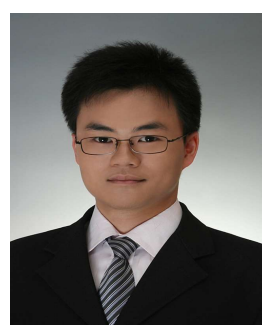

Gong Chen received his Ph.D. in Biomedical Engineering from National University of Singapore, Singapore in 2016. He is now a research fellow in the Singapore Institute for Neurotechnology (SIANPSE), National University of Singapore, Singapore. His current research interests include rehabilitation exoskeleton robot systems, compliant actuator and control theory.

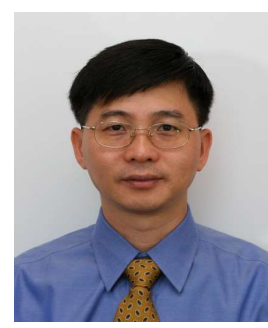

Haoyong Yu (M'10) received the Ph.D. degree in Mechanical Engineering from Massachusetts Institute of Technology, Cambridge, Massachusetts, USA, in 2002. He was a Principal Member of Technical Staff at DSO National Laboratories, Singapore, until 2002. He is currently an Associate Professor with the Department of Biomedical Engineering and a Principal Investigator of the Singapore Institute of Neurotechnology (SINAPSE) at National University of Singapore. His areas of research include medical robotics, rehabilitation engineering and assistive technologies, system dynamics and control.

Dr. Yu received the Outstanding Poster Award at the IEEE Life Sciences Grand Challenges Conference 2013. He served on a number of IEEE Conference Organizing Committees. 